\title{
MATHEMATICAL MODELLING OF AN INNOVATIVE UNMANNED AIRSHIP FOR ITS CONTROL LAW DESIGN
}

\author{
M. Battipede, ${ }^{1}$ M. Lando, ${ }^{1}$ and P. Gili ${ }^{1}$ \\ ${ }^{1}$ Aeronautical and Space Department, Politecnico di Torino, ITALY, \\ $\{$ manuela.battipede, marco.lando,piero.gili $\} @$ polito.it $^{*}$
}

\begin{abstract}
The paper is concerned with the dynamic modelling of the unconventional remotelypiloted Lighter-Than-Air vehicle patented by Nautilus S.p.A. and the Polytechnic of Turin. The airship mathematical model is based on a 6 degree-of-freedom nonlinear model referring to the basic Newtonian mechanics. Emphasis is placed on those innovative and peculiar aspects of the dynamic modelling, such as aerodynamics, buoyancy and inertial features.
\end{abstract}

keywords: airship, dynamic modelling, flight simulation

\section{Introduction}

An innovative remotely-piloted airship has been designed and patented by Nautilus S.p.A. and the Polytechnic of Turin, and equipped with high precision sensors and communication devices by Galileo Avionica and Selex Communication. The object of this project is a low-speed, low-altitude advanced unmanned platform, named Elettra Twin Flyers, which should be employed for reconnaissance, monitoring and telecommunication purposes both in military and civil area. The development of a refined Flight Simulator [1] is essential to support the whole design process of this innovative unmanned airship and its subsequent marketing, as well as to provide a valid platform for the pilot training. In particular, the main task of the Flight Simulator is to assist the airship design process from the early stages, in which it is necessary to evaluate the global dynamic behavior of the vehicle, up to the more advanced phases, in which the single components and subsystems have to be correctly analyzed, dimensioned and integrated in the final product. To obtain a reliable Flight

*Paper written with financial support of Nautilus S.p.A.

Please use the following format when citing this chapter:

Battipede, M., Lando, M., and Gili, P., 2006, in IFIP International Federation for Information Processing, Volume 202, Systems, Control, Modeling and Optimization, eds. Ceragioli, F., Dontchev, A., Furuta, H., Marti, K., Pandolfi, L., (Boston:

Springer), pp. 31-42. 
Simulator it is essential to rely upon a mathematical model, which describes all the peculiar features of the airship from the flight mechanics point of view.

\section{Airship characteristics}

The new concept airship presented in Figure 1 features a double-hull architecture with a central plane housing structure, propellers, on-board energetic system and payload. This unconventional airship does not use aerodynamic control surfaces, therefore, the primary command system is based on six propellers, moved by electrical motors, suitably set in order to produce the desired forces and moments, necessary to control and maneuver the airship both in hovering and forward flight. In particular, two vertical propellers provide the vertical thrust for climbing, descent and pitching maneuvers, while four thrust-vectoring propellers mounted on rotating arms allow to control the lateral-directional attitude of the airship. The lift is basically generated by a hybrid system consisting of aerostatic lift, the buoyancy, provided by the helium inside the hulls, and the vertical thrust given by the vertical propellers. In forward flight, the buoyancy is boosted by the aerodynamic lift developed by the double fuse-shaped body of the airship. In addition, this airship is equipped with two ballonets, one for each hull, which can be blown up with air and deflated, respectively during the descent and climb operations, in order to handle altitude variations without losing helium from the hulls and avoid any significant change in the hull shape [2].

\section{Mathematical model: general assumptions}

The airship mathematical model is based on a six-DOF non linear dynamic model [3], in which the airship is treated as a rigid body without aeroelastic effects and symmetric with respect to the center-line vertical plane $\mathrm{XZ}$. In particular, the model is described by the Newtonian non linear equations of motion, which are expressed through 12 ordinary differential equations. This 12-state formulation basically follows the standard dynamic modelling of the conventional aircraft [4] and assumes that the Earth is fixed in space and its curvature is neglected. However, the presence of aerostatic lift provided by a huge gas volume and the large volume of air displaced by the airship motion give rise to significant additions to the familiar aircraft equations of motion, such as the buoyancy force $B$ and the apparent mass and inertia terms $M_{a p p}$.

These equations of motion are referred to the XYZ body-axes reference frame fixed in the Center of Gravity (CG) of the airship. Due to the airship symmetry, both the $\mathrm{CG}$ and the Center of Buoyancy (CB) lie in the $\mathrm{XZ}$ plane and their coordinates are evaluated in the body-fixed reference frame $O_{X Y Z}$, as shown in Figure 2. Specifically, the CG position $\left(a_{x}, a_{y}, a_{z}\right)$ is referred to the geometric point $O$ fixed on the central plane in between the two vertical propellers, while 
the $\mathrm{CB}$ coordinates $\left(b_{x}, b_{y}, b_{z}\right)$ are related to the $\mathrm{CG}$ position by means of the following expression:

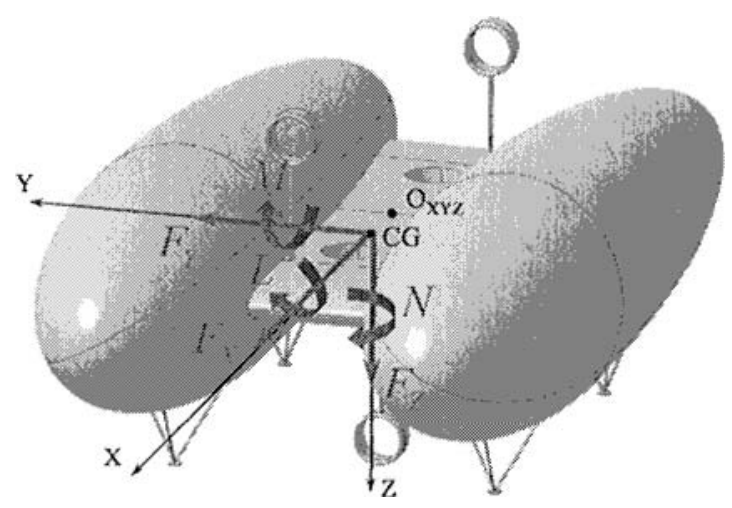

Figure 1. Force \& moment system for the Nautilus unmanned airship

$$
\bar{b}=\left[\begin{array}{l}
x_{C B}-a_{x} \\
y_{C B}-a_{y} \\
z_{C B}-a_{z}
\end{array}\right] \equiv\left[\begin{array}{l}
b_{x} \\
b_{y} \\
b_{z}
\end{array}\right]
$$

where $x_{C B}, y_{C B}$ and $z_{C B}$ are the $\mathrm{CB}$ coordinates with respect to $O_{X Y Z}$, which depend on some flight parameters and are gathered into look-up tables for different flight conditions. In this way, they can be evaluated at each time step of the numerical integration of the equations of motion.

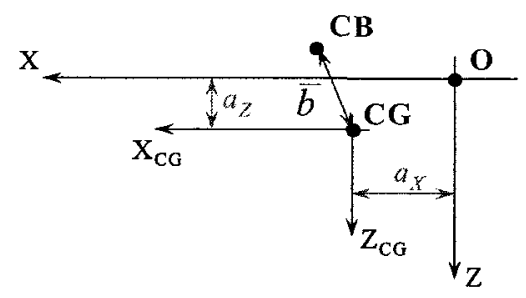

Figure 2. Scheme of the airship reference points

The general scheme of the airship model is illustrated in Figure 3. The complete state vector $\bar{x}$ consists of twelve elements: 


$$
\bar{x}=\left[\begin{array}{llllllllllll}
u & v & w & p & q & r & \varphi & \theta & \psi & N & E & H
\end{array}\right]^{T}
$$

where $(u, v, w)$ are the linear velocities, $(p, q, r)$ are the angular velocities, $(\varphi, \theta, \psi)$ are the Euler angles which define the attitude of the airship relatively to the Earth, while $(N, E, H)$ are the coordinates defining the North-East-Up position of the airship relatively to the Earth. These twelve state variables can be obtained by integrating their time-derivatives with respect to time through the Runge-Kutta numerical integration method. Moreover, the state variables need to be coupled back to all the force and moment equations, as well as to the equations of motion themselves in order to compute their time-derivatives. The inputs of the dynamic model consist of the rotational speeds $n_{p r}$ of all the six propellers and the orientation angles $\delta_{p r}$ of the four thrust-vectoring propellers. These inputs feed the propulsion system and generate the desired propulsive forces and moments needed to maneuver the airship. In particular, these signals are generated by the pilot acting on a joystick and two throttles. Successively, the pilot commands are pre-processed and re-allocated by the Control Allocation System [5] modelled in the Flight Control Computer and, finally, are filtered by first order transfer functions that account for the actuator dynamics.

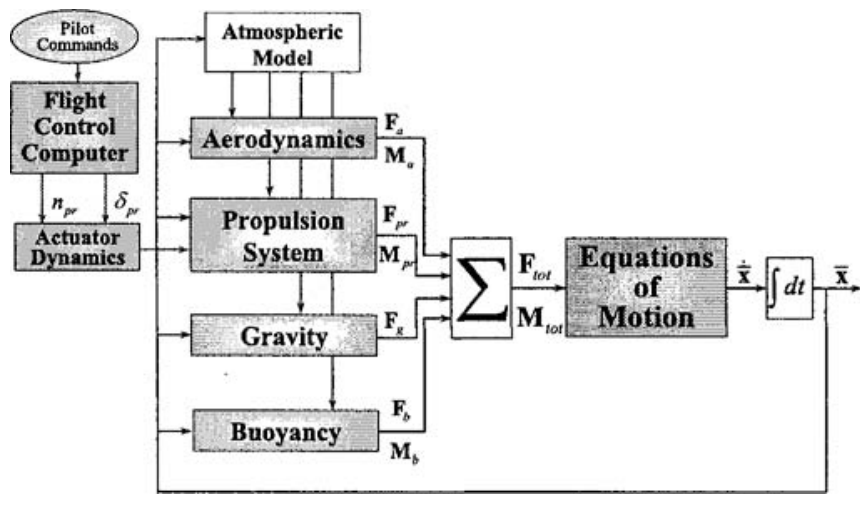

Figure 3. General scheme of the airship model

\section{Equations of motion}

The airship equations of motion are expressed in the non linear state-space format and consist of six force and moment equations, three kinematic equations 
and three navigation equations. The axial, side and normal force equations and the rolling, pitching and yawing moments equations with respect to the $\mathrm{CG}$ reference frame are computed by implementing the Newton's second law of motion for each degree of freedom. In a matricial form:

$$
M \dot{\bar{x}}_{v}=\bar{F}_{d}+\bar{F} \Rightarrow \dot{\bar{x}}_{v}=M^{-1}\left(\bar{F}_{d}+\bar{F}\right)
$$

where $\dot{\bar{x}}_{v}=\left[\begin{array}{llllll}\dot{u} & \dot{v} & \dot{w} & \dot{p} & \dot{q} & \dot{r}\end{array}\right]^{T}$ is the partial state vector of linear and angular accelerations, $M$ is the total mass matrix, $\bar{F}_{d}$ and $\bar{F}$ represent respectively the dynamic contributions and the external contributions depending on aerodynamics, static buoyancy, propulsion system and gravitational force:

$$
\bar{F}_{d}=\left[\begin{array}{c}
-m_{z} w q+m_{y} r v \\
-m_{x} u r+m_{z} p w \\
-m_{y} v p+m_{x} q u \\
-\left(J_{z}-J_{y}\right) r q+J_{y z}\left(q^{2}-r^{2}\right)+J_{z x} p q-J_{x y} p r \\
-\left(J_{x}-J_{z}\right) p r-J_{y z} p q+J_{z x}\left(r^{2}-p^{2}\right)+J_{x y} q r \\
-\left(J_{y}-J_{x}\right) q p+J_{y z} p r-J_{z x} q r+J_{x y}\left(p^{2}-q^{2}\right)
\end{array}\right]
$$

$F_{X, Y, Z}$ are the generic force components in the $C G$ reference frame, while $L, M$, $N$ are the generic moments around the $X-, Y-$ and $Z$-body axes respectively, as shown in Figure 1. The terms $m_{x, y, z}$ and $J_{x, y, z, x y, z x, y z}$ represent the airship inertial elements of the total mass matrix $M$ :

$$
M=M_{i}+M_{g}-M_{a p p}
$$

The airship inertial data, in fact, have been computed on the assumption that $M$ has to account for three contributions [6]: 1) the mass and inertia terms $M_{i}$ of all the airship structure components; 2) the inertial properties $M_{g}$ of the gas inside hulls and ballonets; 3 ) the apparent mass and inertia terms $M_{a p p}$ arising from the large volumes of air displaced by the airship motion, especially in non stationary conditions. The inertial characteristics of airship structure components and gas are expressed as follows: 


$$
M_{i}+M_{g}=\left[\begin{array}{cccccc}
m & 0 & 0 & 0 & 0 & 0 \\
0 & m & 0 & 0 & 0 & 0 \\
0 & 0 & m & 0 & 0 & 0 \\
0 & 0 & 0 & I_{x x} & -I_{x y} & -I_{x z} \\
0 & 0 & 0 & -I_{x y} & I_{y y} & -I_{y z} \\
0 & 0 & 0 & -I_{x z} & -I_{y z} & I_{z z}
\end{array}\right]+\left[\begin{array}{cccccc}
m_{g} & 0 & 0 & 0 & 0 & 0 \\
0 & m_{g} & 0 & 0 & 0 & 0 \\
0 & 0 & m_{g} & 0 & 0 & 0 \\
0 & 0 & 0 & I_{x x_{g}} & -I_{x y_{g}} & -I_{x z_{g}} \\
0 & 0 & 0 & -I_{x y_{g}} & I_{y y_{g}} & -I_{y z_{g}} \\
0 & 0 & 0 & -I_{x z_{g}} & -I_{y z_{g}} & I_{z z_{g}}
\end{array}\right]
$$

The apparent mass and inertial terms are defined by the following matrix:

$$
M_{a p p}=\left[\begin{array}{cccccc}
X_{\dot{u}} & X_{\dot{v}} & X_{\dot{w}} & X_{\dot{p}} & X_{\dot{q}} & X_{\dot{r}} \\
Y_{\dot{u}} & Y_{\dot{v}} & Y_{\dot{w}} & Y_{\dot{p}} & Y_{\dot{q}} & Y_{\dot{r}} \\
Z_{\dot{u}} & Z_{\dot{v}} & Z_{\dot{w}} & Z_{\dot{p}} & Z_{\dot{q}} & Z_{\dot{r}} \\
L_{\dot{u}} & L_{\dot{v}} & L_{\dot{w}} & L_{\dot{p}} & L_{\dot{q}} & L_{\dot{r}} \\
M_{\dot{u}} & M_{\dot{v}} & M_{\dot{w}} & M_{\dot{p}} & M_{\dot{q}} & M_{\dot{r}} \\
N_{\dot{u}} & N_{\dot{v}} & N_{\dot{w}} & N_{\dot{p}} & N_{\dot{q}} & N_{\dot{r}}
\end{array}\right]
$$

The effects of the gas $M_{g}$ are estimated by modelling the gas/air mass inside hulls and ballonets through the Catia CAD code [7]. In this way, the gas inertial characteristics, such as mass $m_{g}$, gas center-of-gravity position $\left(x_{g}, y_{g}, z_{g}\right)$ and inertia moments $\left(J_{x x_{g}}, J_{y y_{g}}, J_{z z_{g}}\right.$ ) with respect to $O_{X Y Z}$, can be evaluated and gathered into look-up tables in order to compute the gas inertial contributions with respect to the airship $\mathrm{CG}$ reference frame:

$$
\left\{\begin{aligned}
I_{x x_{g}} & =J_{x x_{g}}-m_{g}\left[\left(z_{g}-a_{z}\right)^{2}+\left(y_{g}-a_{y}\right)^{2}\right] \\
I_{y y_{g}} & =J_{y y_{g}}-m_{g}\left[\left(x_{g}-a_{x}\right)^{2}+\left(z_{g}-a_{z}\right)^{2}\right] \\
I_{z z_{g}} & =J_{z z_{g}}-m_{g}\left[\left(x_{g}-a_{x}\right)^{2}+\left(y_{g}-a_{y}\right)^{2}\right]
\end{aligned}\right.
$$

On the contrary, the products of inertia $I_{x y_{g}}, I_{x z_{g}}, I_{y z_{g}}$ in the matrix $M_{g}$ are assumed to be equal to zero.

The apparent mass and inertial effects $M_{a p p}$ may be considered as added forces and moments, therefore, they can be described by the dimensional derivatives of aerodynamic forces and moments with respect to linear and angular acceleration perturbations, i.e. $X_{\dot{u}}=\partial F_{X_{a}} / \partial \dot{u}$ or $L_{\dot{p}}=\partial L / \partial \dot{p}$. The non dimensional coefficients of these derivatives have been calculated with respect to the $O_{X Y Z}$ reference frame by using NSAERO [8], a multi-block computational fluid dynamics code, which solves the Navier-Stokes equations including also the viscous effects. Before being included into $\mathrm{Eq}$. (6), however, the matrix $M_{a p p}$ must be rewritten with respect to the $\mathrm{CG}$ reference frame. This translation can be done on the assumptions that the fluid kinetic energy does not depend on the reference frame: 


$$
\frac{1}{2} \bar{x}_{v_{C G}}^{T} M_{a p p_{C G}} \bar{x}_{v_{C G}}=\frac{1}{2} \bar{x}_{v_{O}}^{T} M_{a p p_{O}} \bar{x}_{v_{O}}
$$

and the velocity vector $\bar{x}_{v_{O}}$ of the body axis origin $O$ can be related to the velocity vector $\bar{x}_{v_{C G}}$ of the airship CG:

$$
\bar{x}_{v_{O}}=\left[\begin{array}{c|c}
I & A \\
\hline 0 & I
\end{array}\right]^{-1} \bar{x}_{v_{C G}} \equiv\left[\begin{array}{c|c}
I & -A \\
\hline 0 & I
\end{array}\right] \bar{x}_{v_{C G}}
$$

where $A$ is the rotational matrix from the $O_{X Y Z}$ to the $C G$ reference frame and it is made up by the airship CG coordinates $\left(a_{x}, a_{y}, a_{z}\right)$ :

$$
A=\left[\begin{array}{ccc}
0 & a_{z} & -a_{y} \\
-a_{z} & 0 & a_{x} \\
a_{y} & -a_{x} & 0
\end{array}\right]
$$

Finally, the apparent mass matrix in the $\mathrm{CG}$ reference frame will be:

$$
M_{a p p_{C G}}=\left[\begin{array}{c|c}
I & 0 \\
\hline A & I
\end{array}\right] M_{a p p_{O}}\left[\begin{array}{c|c}
I & -A \\
\hline 0 & I
\end{array}\right]
$$

In order to solve the equations of motion of Eq. (3), it is also necessary to know the attitude, defined by the Euler angles $(\varphi, \theta, \psi)$, and the altitude $H$ of the airship, because some contributions to the external forces and moments $\bar{F}$ of Eq. (5) depend upon these variables. Moreover, it is useful to evaluate the coordinates of the vehicle with respect to the Earth-fixed reference frame to be able to simulate navigational tasks. Firstly, the three kinematic equations defining the airship attitude rates are respectively:

$$
\left\{\begin{array}{l}
\dot{\varphi}=p+\dot{\psi} \sin \theta \\
\dot{\theta}=q \cos \varphi-r \sin \varphi \\
\dot{\psi}=\frac{q \sin \varphi+r \cos \varphi}{\cos \theta}
\end{array}\right.
$$

Secondly, the three navigation equations computing the Earth-relative velocities are defined by the following expressions:

$$
\left\{\begin{aligned}
\dot{N}= & u \cos \theta \cos \psi+v(\sin \varphi \sin \theta \cos \psi-\cos \varphi \sin \psi)+ \\
& +w(\sin \varphi \sin \psi+\cos \varphi \sin \theta \cos \psi) \\
\dot{E}= & u \cos \theta \sin \psi+v(\sin \varphi \sin \theta \sin \psi+\cos \varphi \cos \psi)+ \\
& +w(\cos \varphi \sin \theta \sin \psi-\sin \varphi \cos \psi) \\
\dot{H}= & u \sin \theta-(v \sin \varphi+w \cos \varphi) \cos \theta
\end{aligned}\right.
$$




\section{Aerodynamics and buoyancy}

The airship aerodynamic modelling is based on the six aerodynamic coefficients $\left(C_{X_{a}}, C_{Y_{a}}, C_{Z_{a}}, C_{l_{a}}, C_{m_{a}}, C_{n_{a}}\right.$ ) and the eighteen damping adimensional derivatives $\left(C_{X_{p, q, r}}, C_{Y_{p, q}, r}, C_{Z_{p, q, r}}, C_{l_{p, q, r}}, C_{m_{p, q, r}}, C_{n_{p, q, r}}\right)$, which have been computed in the $O_{X Y Z}$ reference frame by using the NSAERO code. These coefficients depend on some flight parameters, such as the airspeed, the angle of attack $\alpha$ and the sideslip angle $\beta$. In particular, they are estimated on a rough grid for $0 \leq \alpha \leq 90^{\circ}$ and $0 \leq \beta \leq 180^{\circ}$, at different airspeeds and at zero altitude. These data are successively processed, interpolated and extrapolated into thicker grids and more extended ranges of the airspeed, the angle of attack $\left(-90^{\circ} \leq \alpha \leq+90^{\circ}\right)$, and the sideslip angle $\left(-180^{\circ} \leq \beta \leq+180^{\circ}\right)$, in order to obtain suitable 3-D look-up tables, as shown in Figure 4, in which the coefficient signs are opportunely changed according to the axis conventions. This 360-degree aerodynamic modelling allows the airship dynamic model to handle either hovering or any other forward flight condition and avoid discontinuities in passing from hovering to forward flight.

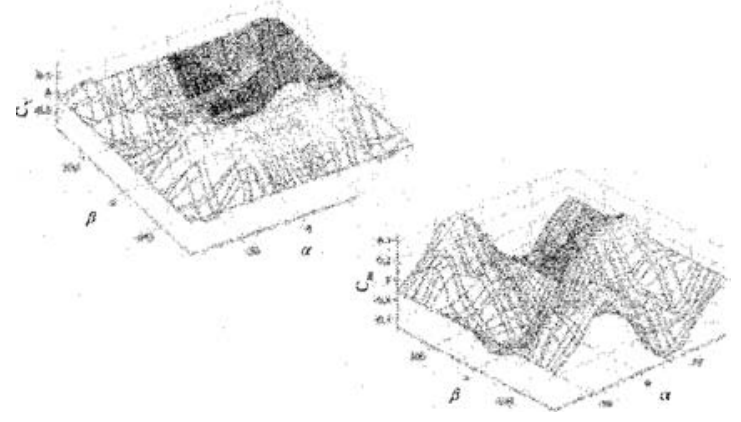

Figure 4. Aerodynamic modelling through Look-Up Tables

Finally, the aerodynamic forces and moments can be expressed in the standard notation as follows:

$$
\left\{\begin{array}{l}
F_{X_{a}}=\left(C_{X_{a}}+C_{X_{p}} \hat{p}+C_{X_{q}} \hat{q}+C_{X_{r}} \hat{r}\right) \cdot 0.5 \rho U^{2} V^{2 / 3} \\
F_{Y_{a}}=\left(C_{Y_{a}}+C_{Y_{p}} \hat{p}+C_{Y_{q}} \hat{q}+C_{Y_{r}} \hat{r}\right) \cdot 0.5 \rho U^{2} V^{2 / 3} \\
F_{Z_{a}}=\left(C_{Z_{a}}+C_{Z_{p}} \hat{p}+C_{Z_{q}} \hat{q}+C_{Z_{r}} \hat{r}\right) \cdot 0.5 \rho U^{2} V^{2 / 3} \\
L_{a}=\left(C_{l_{a}}+C_{l_{p}} \hat{p}+C_{l_{q}} \hat{q}+C_{l_{r}} \hat{r}\right) \cdot 0.5 \rho U^{2} V^{2 / 3} D \\
M_{a}=\left(C_{m_{a}}+C_{m_{p}} \hat{p}+C_{m_{q}} \hat{q}+C_{m_{r}} \hat{r}\right) \cdot 0.5 \rho U^{2} V^{2 / 3} l \\
N_{a}=\left(C_{n_{a}}+C_{n_{p}} \hat{p}+C_{n_{q}} \hat{q}+C_{n_{r}} \hat{r}\right) \cdot 0.5 \rho U^{2} V^{2 / 3} D
\end{array}\right.
$$


where $\hat{p}=b p / 2 U, \hat{q}=c q / 2 U, \hat{r}=b r / 2 U$ are the adimensional angular velocities, $\rho$ is the air density, $U$ is the free-stream airspeed, $V$ is the airship volume, $D$ is the hull diameter and $l, c, b$ are reference lengths. Obviously, these aerodynamic forces need to be properly translated in the CG reference frame through the rotational matrix $A$, before being integrated in $\mathrm{Eq}$. (5):

$$
\left\{\begin{array}{c}
\bar{F}_{a} \\
\bar{M}_{a}
\end{array}\right\}_{C G}=\left[\begin{array}{l|l}
I & 0 \\
\hline A & I
\end{array}\right]\left\{\begin{array}{c}
\bar{F}_{a} \\
\bar{M}_{a}
\end{array}\right\}_{O}
$$

The main contribution to the airship lift is supplied by the aerostatic buoyancy provided by the helium inside the hulls. The two inner ballonets can be blown up with air and deflated to handle altitude variations. In particular, air is initially released from ballonets during climb up to the plenitude altitude $H_{\max }$, that is the altitude defined before each mission according to the amount of helium contained in the hulls and to which the gas is completely expanded filling the hulls themselves. Beyond $H_{\max }$, helium has to be released from hulls causing the reduction of buoyancy $B$. Differently, during descent ballonets are blown up by the on-board pneumatic system [2]. Assuming constant pressure and temperature inside the hulls, $B$ does not vary from the ground to the plenitude altitude. Beyond $H_{\max }, B$ decreases proportionally to the air specific weight according to the second relation of Eq. (14):

$$
\begin{cases}B=V_{b} \Lambda_{a i r_{0}}\left(1-\frac{1}{\varepsilon_{H e}}\right) & H \leq H_{\max } \\ B=V_{b} \Lambda_{a i r_{0}} \frac{p_{a} T_{K_{0}}}{p_{a_{0}} T_{K}}\left(1-\frac{1}{\varepsilon_{H e}}\right) & H>H_{\max }\end{cases}
$$

where $V_{b}$ is the helium volume, $\Lambda_{a i r_{0}}$ is the specific weight of air at zero altitude, $\varepsilon_{H e}$ is the air/helium specific weight ratio, $p_{a}$ and $T_{K}$ are respectively the pressure and the temperature of the atmosphere.

The buoyancy action is applied in CB, whose location, defined in Eq. (1), changes with the altitude depending on the amount of air contained in the ballonets. The presence of ballonets and their functioning are mathematically modelled through the variations $\Delta x$ and $\Delta z$ of the $C B$ position with respect to the $O_{X Y Z}$ reference frame. The lateral shift $\Delta y$ of the buoyancy is assumed to be negligible, while $\Delta x$ and $\Delta z$ have been estimated through the Catia CAD code by considering an initial air-helium subdivision of the hulls and, successively, varying the ballonet volume to simulate the altitude variation [9]. Finally, the $\Delta x$ and $\Delta z$ variations, needed to evaluate the CB coordinates at each time step of the simulation, are gathered into look-up tables as a function of the altitude $H$ and the plenitude altitude $H_{\max }$. Analogously to the gravitational force $m g$, the buoyancy $B$ acts along the Z-Earth axis ( $N E D$ inertial reference frame), thus, it needs to be transferred in the CG body reference frame through the Euler rotational matrix $\{E\}$ : 


$$
\begin{gathered}
\bar{F}_{b_{C G}}=\{E\}\left\{\begin{array}{lll}
0 & 0 & -B
\end{array}\right\}_{N E D}^{T} \\
\{E\}=\left[\begin{array}{ccc}
\cos \theta \cos \psi & \cos \theta \sin \psi & -\sin \theta \\
-\cos \varphi \sin \psi+\sin \varphi \sin \theta \cos \psi & \cos \varphi \cos \psi+\sin \varphi \sin \theta \sin \psi & \sin \varphi \cos \theta \\
\sin \varphi \sin \psi+\cos \varphi \sin \theta \cos \psi & -\sin \varphi \cos \psi+\cos \varphi \sin \theta \sin \psi & \cos \varphi \cos \theta
\end{array}\right]
\end{gathered}
$$

The buoyancy moments $\bar{M}_{b_{C G}}$ are then computed by means of the CB coordinates $\left(b_{x}, b_{y}, b_{z}\right): \bar{M}_{b_{C G}}=\bar{b} \wedge \bar{F}_{b_{C G}}$.

\section{Propulsive actions}

The thrust $T$ of all the six propellers is modelled through the first Renard formula $T=\tau \rho \omega^{2} R^{4}$, where $\rho$ is the air density, $R$ is the radius and $\omega$ is the angular rate of the propellers. $\tau$ represents the thrust coefficient, which is a function of the propeller working point $\gamma=U_{a x} / \omega R$, where $U_{a x}$ is the axial airspeed component at the propeller disc along its rotational axis. The first command input $n_{p r}$ acts on the propeller angular rate $\omega$. Figure 5 shows the disposition of the six propellers in the airship central plane. In particular, there are four thrust-vectoring propellers in asymmetrical positions: front up $(F U)$, front down (FD), rear up (RU), rear down (RD); while the two vertical ducted propellers are in the fore (VF) and aft (VA) part of the central plane. The orientation $\delta$ of each of the four thrust-vectoring propellers is generated by the second command input $\delta_{p r}$.

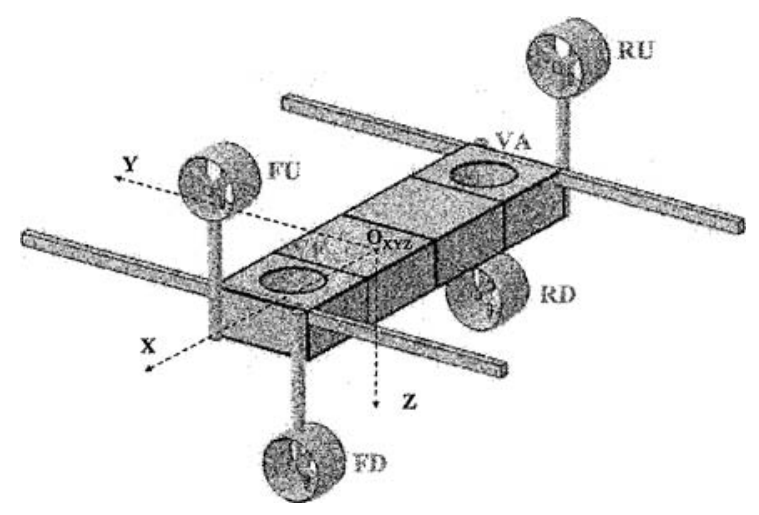

Figure 5. Scheme of the primary control system 
The total propulsive forces and moments in the $O_{X Y Z}$ reference frame are carried out by adding up the contributions of all the propellers:

$$
\begin{aligned}
& \left\{\begin{array}{l}
F_{X_{p r}}=T_{F U} \cos \delta_{F U}+T_{R D} \cos \delta_{R D}+T_{R U} \cos \delta_{R U}+T_{F D} \cos \delta_{F D} \\
F_{Y_{p r}}=T_{F U} \sin \delta_{F U}+T_{R D} \sin \delta_{R D}+T_{R U} \sin \delta_{R U}+T_{F D} \sin \delta_{F D} \\
F_{Z_{p r}}=-T_{V T}-T_{V A}
\end{array}\right. \\
& \bar{M}_{p r}=\bar{F}_{p r} \wedge \bar{l}_{p r} \equiv\left[\begin{array}{ccc}
0 & F_{Z_{p r}} & -F_{Y_{p r}} \\
-F_{Z_{p r}} & 0 & F_{X_{p r}} \\
F_{Y_{p r}} & F_{X_{p r}} & 0
\end{array}\right]\left\{\begin{array}{c}
x_{p r_{0}} \\
y_{p r_{0}} \\
z_{p r_{0}}
\end{array}\right\}
\end{aligned}
$$

where $\bar{l}_{p r}$ represents the position vector of each propeller with respect to $O_{X Y Z}$. Successively, $\bar{F}_{p r}$ and $\bar{M}_{p r}$ have to be transferred in the CG reference frame by using an expression analogous to Eq. (17).

\section{Conclusions}

The airship mathematical model previously described was implemented in the Matlab/Simulink environment and, undoubtedly, represented the first step in the development of a refined Flight Simulator, whose availability is definitely essential for the design, test and implementation of the most suitable flight control laws for the innovative Nautilus airship. The general scheme of this Flight Simulator consists basically of two entities, the pilot station and the remote station, which are clearly illustrated in Figure 6.

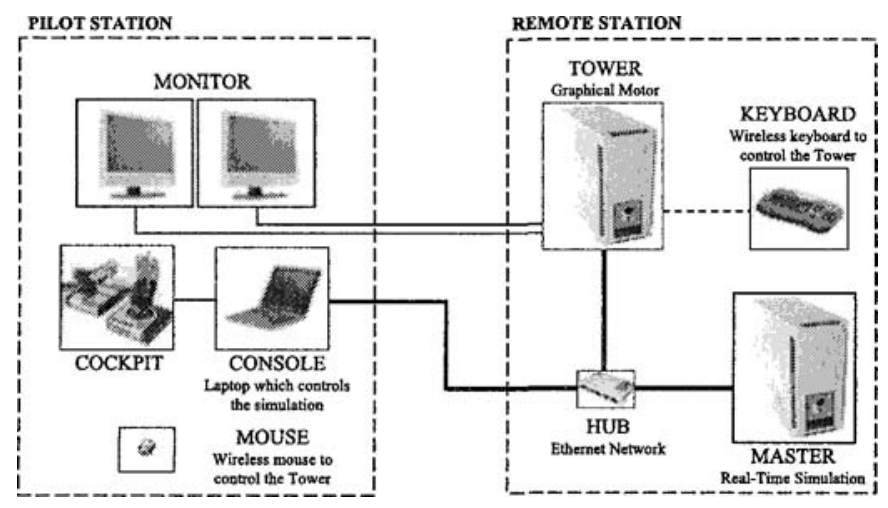

Figure 6. General scheme of the Flight Simulator 


\section{References}

[1] M. Battipede, P.A. Gili, M. Lando. Ground Station and Flight Simulator for a RemotelyPiloted Non Conventional Airship. In AIAA Guidance, Navigation and Control Conference. Reston, VA, 2005.

[2] M. Battipede, M. Lando, P.A. Gili, P. Vercesi. Peculiar Performance of a New LighterThan-Air Platform for Monitoring. In AIAA Aviation Technology, Integration and Operation Forum. Reston, VA, 2004.

[3] G.A. Khoury, J.D. Gillett. Airship Technology. Cambridge University Press, 1999.

[4] B.L. Stevens, F.L. Lewis. Aircraft Control and Simulation. John Wiley \& Sons, Inc., New York, 1992.

[5] M. Battipede, P.A. Gili, M. Lando. Control Allocation System for an Innovative RemotelyPiloted Airship. In AIAA Atmospheric Flight Mechanics Conference. Reston, VA, 2004.

[6] P.G. Thomasson. Equations of Motion of a Vehicle in a Moving Fluid. Journal of Aircraft. Vol. 37, 4:630-639, 2000.

[7] CATIA V5R12. 3-D CAD Software. Dassault Systèmes S.A., 2003.

[8] NSAERO. Advanced Fluid Dynamics Software. Analytical Methods, Inc, Redmond, WA.

[9] M. Battipede, P.A. Gili, M. Lando, L. Massotti. Flight Simulator for the Control Law Design of an Innovative Remotely-Piloted Airship. In AIAA Modeling and Simulation Technologies Conference. Reston, VA, 2004. 